\title{
Improving Access to Health and Gender-based Violence Services for Female Entertainment Workers in Cambodia: Findings From the Mobile Link Randomized Controlled Trial
}

\section{Carinne Brody}

Touro University California

Pheak Chhoun

KHANA Center for Population Research

Sovannary Tuot

KHANA Center for Population Health Research

Anne E. Fehrenbacher

University of California Los Angeles

\section{Alexander Moran}

University of California Los Angeles

\section{Dallas Swendeman}

University of California Los Angeles

Siyan Yi ( $\nabla$ ephsyi@nus.edu.sg )

KHANA Center for Population Health Research https://orcid.org/0000-0002-3045-5386

\section{Research}

Keywords: Female sex workers, HIV, Linkage to services, Sexual and reproductive health, Gender-based violence, Low- and middle-income country

Posted Date: January 27th, 2021

DOl: https://doi.org/10.21203/rs.3.rs-154027/v1

License: (c) (1) This work is licensed under a Creative Commons Attribution 4.0 International License. Read Full License 


\section{Abstract}

Background: Female entertainment workers (FEWs) in Cambodia experience a greater prevalence of human immunodeficiency virus (HIV), other sexually transmitted infections (STIs), psychological distress, substance use, and gender-based violence (GBV) than the general population. Reaching FEWs with health education and linking them to services has been difficult because of their hidden and stigmatized status. This study evaluates the Mobile Link intervention, aiming to improve FEWs' health by connecting them to existing HIV, sexual and reproductive health, and GBV services using short message services and voice messages.

Methods: This randomized controlled trial was conducted between March 2018 and June 2019. We employed a stratified random sampling method to select participants from five study sites. Initially, we randomly selected 600 participants from a list of $4000 \mathrm{FEWs}$ by age group and study site and randomized them to intervention or standard care control arms. The primary outcome measures included self-reported HIV and STI testing, condom use, and contraceptive use. We also measured secondary outcomes, including contact with outreach workers, escorted referral services use, forced drinking, and GBV experiences. Intervention effects were modeled using repeated measures, multilevel mixed-effects logistic regression.

Results: We included 218 FEWs in intervention and 170 FEWs in control arms in the per-protocol analyses after removing dropouts. Evidence of positive intervention effects was detected for the following secondary outcomes: contacting an outreach worker (group by time 2 AOR 3.29, 95\% Cl 1.28-8.47), receiving an escorted referral (group by time 2 AOR 2.86, 95\% $\mathrm{Cl} 1.09-7.52$; group by time 3 AOR 8.15, 95\% $\mathrm{Cl} 1.65-40.25$ ), and never being forced to drink at work (group by time $3 \mathrm{AOR} 3.95,95 \% \mathrm{Cl} 1.62-$ 9.60). No significant differences between intervention and control groups over time were observed for any primary outcomes in the fully adjusted models.

Conclusions: The Mobile Link intervention effectively connected FEWs with outreach workers and escorted referrals. Reductions in forced drinking at work were also significantly more extensive in the intervention group than the control group. Longer-term messaging may increase access to services and impact FEWs' health outcomes in the future.

Trial registration: Clinical trials.gov, NCT03117842 . Registered 31 March 2017, https://clinicaltrials.gov/ct2/show/NCT03117842?term=NCT03117842\&rank=1

\section{Contributions To The Literature}

- This randomized controlled trial is one of few studies to date that have rigorously evaluated interventions using mobile technology for reaching and improving access to health services among hard-to-reach populations in resource-limited settings.

- We developed this trial based on extensive experience and participatory formative studies with female entertainment worker communities and key stakeholders. 
- Lessons learned from this study demonstrated that mobile-health approaches could link hard-toreach populations with necessary health services.

- We identified several barriers to developing and implementing community-based mobile-health interventions that are of good quality, scalable, and sustainable.

\section{Background}

Female entertainment and sex workers experience a greater prevalence of human immunodeficiency virus (HIV), other sexually transmitted infections (STIs) [1, 2], psychological distress [3], substance use [3, 4], and gender-based violence (GBV) $[1,5]$ than the general women population. These health burdens are intensified by social and structural factors, including poverty, gender inequality, discrimination, and stigmatization [1, 2]. In Cambodia, these health trends and associations hold for female entertainment workers (FEWs) who are employed at establishments such as karaoke bars, restaurants, beer gardens, or massage parlors [6, 7]. Approximately one-third of FEWs exchange sex to supplement their income [8]. Transactional sex puts FEWs at high risk for adverse health outcomes, which can threaten their psychological, physical, and family health $[7,8]$.

FEWs in Cambodia often come from poor and rural families, originally migrating to cities at a young age in hopes of earning higher wages to remit to their families [7-9]. FEWs have an HIV prevalence of nearly $10 \%$ and low HIV and STI testing rates and service seeking [10]. They also have low rates of modern contraceptive use and high rates of induced abortion $[8,11]$ and are frequent victims of GBV $[7,12]$.

FEWs face barriers to accessing health services. As an HIV key population in Cambodia, they are eligible to receive free health services provided by non-governmental organizations (NGOs). However, many do not seek services due to stigma and discrimination [10]. The population's health risk profile is further exacerbated by Cambodia's 2008 Law on the Suppression of Human Trafficking and Sexual Exploitation, which bans prostitution. Research indicates a strong association between sex work criminalization and sex workers' increased risk for HIV and STIs, sexual and physical violence, and unprotected sex [13]. In Cambodia, outlawing prostitution has amplified stigma against FEWs and deterred this population from carrying condoms, which police use as evidence of sex work. The law also creates mistrust of police among FEWs, which prevents them from reporting instances of GBV to the police, who frequently raid entertainment establishments and arrest FEWs $[14,15]$. Additionally, the law is associated with a substantial increase in the number of FEWs as more women move from brothels to entertainment venues, where they can continue to exchange sex [6, 7]. In 2008, an estimated number of 13000 FEWs were reported in Cambodia, but by 2018, that number had risen to approximately 40000 [16].

Health interventions using mobile phones - referred to as mobile health (mHealth) - presents a viable solution for connecting hard-to-reach, stigmatized, and criminalized populations such as FEWs to health services. In recent years, mHealth has received widespread attention due to its applicability in lowresource settings. mHealth has been used effectively in low- and middle-income countries to collect and report community health data [17], disperse health education information [17], raise health awareness 
[18], and conduct routine check-ins with patients and trigger follow-ups by nurses [19]. However, knowledge gaps persist in mHealth research. Fewer mHealth interventions have been rigorously evaluated. Much of the existing literature comprises small pilot studies lacking established health indicators and generalizability [20-22]. Additionally, there is a deficiency in mHealth research on interventions targeted towards behavior change and sexual and reproductive health (SRH) [21].

The Mobile Link intervention is an mHealth project aiming to engage and connect young FEWs in Cambodia to prevention, care, and treatment services using automated short message service (SMS) and voice messages (VM). The Mobile Link intervention is based on a behavior-change theory and years of formative research. The intervention aims to reduce FEWs' risk behaviors and increase HIV, STI, SRH, and GBV service utilization. The project's details are described in the formative study and protocol papers, with an overview provided below $[23,24]$. This research strives to fill existing knowledge gaps in mHealth research and provide information on a hard-to-reach, high-risk, and understudied population. This study aims to evaluate the efficacy of the Mobile Link intervention in engaging FEWs, connecting them to existing HIV, SRH, and GBV services, and ultimately improving their health.

\section{Methods}

\section{Trial design and settings}

The Mobile Link intervention study is a multisite 60-week randomized controlled trial (RCT). The trial was conducted in two sites in Phnom Penh and one site each in Banteay Meanchey, Battambang, and Siem Reap. These provinces were selected because of substantial populations of FEWs and high HIV burdens.

\section{Participants}

The intervention's participant inclusion criteria included: (1) working at an entertainment venue in the study sites; (2) being currently sexually active, defined as having engaged in oral, vaginal, or anal sex in the past three months; (3) owning a mobile phone; (4) knowing how to retrieve VM or retrieve and read SMS; (5) self-identifying as a FEW; (6) willing to receive two SMS/VM per week for one year; (7) providing written informed consent; and (8) agreeing to a follow-up visit after six months and 12 months.

\section{Randomization}

Field workers developed a list of more than $4000 \mathrm{FEWs}$ from the five study sites. FEWs in the list were categorized by site and age group (18-24 and 25-30 years old). At each site, 60 FEWs were randomly selected (30 in the age group of 18-24 and 30 in the age group of 25-30) for each arm (300 FEWs in the intervention and 300 FEWs in the control arm) for a total of 600 study participants.

\section{Recruitment}

All participants were recruited in-person at the five study sites by trained Mobile Link lay community health workers. Community health workers provided verbal information to FEWs regarding the Mobile LinKs details because of low literacy rates in this population. Eligible FEWs signed the informed consent 
form and provided community health workers with mobile numbers for all of their subscriber identification module (SIM) cards and indicated which SIM they used most often. Recruited FEWs were assigned a unique identification number to protect their privacy and blind the researchers from their treatment arm assignment.

At the midline, the data collection team recruited additional study participants to replace those who were lost to follow-up by randomly selecting FEWs from the same site and age group on the master list, excluding those that had ever been selected. These replacement participants had the opportunity to participate in the second 30 weeks of the intervention period. We considered participants having at least two survey assessments to be active participants in the study.

\section{Patient and public involvement}

This clinical trial was developed after months of iterative qualitative data collection with FEWs, including 27 focus group discussions, nine in-depth interviews, and two validation workshops. During this period, participants gave researchers guidance and feedback on the design and delivery of the intervention and recruitment and data collection procedures. Dissemination activities involve extensive focus group discussions, in-depth interviews, and a final validation workshop.

\section{Intervention}

The Mobile Link intervention was informed by both behavior change theories and extensive formative research. The intervention provided FEWs with information, resources, and reminders. By utilizing an SMS/VM platform, these services were provided in a convenient, accessible, inexpensive, and confidential manner. Therefore, we theorized that this delivery mechanism would improve FEWs' knowledge of existing resources, risks, risk behaviors, and positive attitudes related to these topics. Increasing knowledge and positive attitudes will contribute to skill acquisition and positive behavior change.

We conducted a series of formative research activities using participatory methods to create appropriate and relevant health-related messages for FEWs and inform the intervention's development. The formative research process occurred over six months. We collected data through focus group discussions (FGDs), in-depth interviews (IDIs), and key informant interviews (KIIs) with the venue- and non-venue-based FEWs in addition to outreach workers and field staff that routinely work with this population [23]. Findings from the formative research revealed that FEWs were generally knowledgeable about HIV and STI prevention and transmission. However, they faced many structural barriers to optimal health, such as pressure to drink alcohol at work and complicated dynamics of negotiating condom use with clients in a criminalized environment $[25,26]$. Furthermore, we found that many FEWs faced barriers to accessing medical care and services due to stigma, discrimination, and mistreatment from healthcare workers.

Development of the message-based intervention was conducted with the support of local partners, InSTEDD iLab and the Women's Media Center (WMC). InSTEDD developed the mobile platform for interactive message delivery and data management using an open-source software program. The WMC 
helped translate messages into Khmer and tailor the contents to be specific, relevant, and engaging, given the cultural context. Example messages included can be found in a previously published paper [23].

After development, the intervention underwent a four-week pilot in which 50 FEWs from each study site were randomly selected. The purpose of the pilot was to test whether the platform functioned well with the intervention design and whether the intervention was feasible and acceptable for the participants.

The central components of the Mobile Link intervention were the SMS and VM messages, containing health information, and referral linkage information to health services and resources. From the formative research process, 180 messages were designed covering ten health themes identified as the most important by participants. The health themes covered the following topics: cervical cancer, contraception, general health information, HIV and STI transmission and prevention, miscarriage, pregnancy, alcohol use at work, pregnancy termination, hygiene and vaginal health, and GBV. A message was delivered twice a week for ten weeks, and the message from each topic area was repeated every ten weeks for 60 weeks. The health messages were framed using rights-based and health promotion frameworks. Participants could choose to receive the messages in an SMS or VM form that worked with simple and smartphone devices. Those who chose the SMS message option could further personalize their choice by selecting Khmer characters or Romanized Khmer. Each health topic message was followed by a message providing FEWs with the option to be linked to an outreach worker. Participants who selected this option were called by the Mobile Link's staff, who would provide individualized information via telephone or face-to-face and, if needed, would escort the participant to services.

The control for this study received the existing standard care. Standard care included face-to-face counseling, free HIV and STI testing and condoms, and clinic phone numbers and hotline phone numbers with a toll-free help-line for clients staffed by trained counselors. The group did not receive the healthrelated SMS/VM component. However, they will receive a "check-in" SMS or VM between baseline and midline and another between midline and endline to stay in touch with the participants and remind them of the interview appointments.

\section{Outcomes and measures}

The primary outcome measures of the Mobile Link intervention were: (1) HIV testing, (2) STI testing when experiencing symptoms, (3) contraceptive use, (4) always use condoms with non-paying partners, and (5) always use condoms with paying partners. The secondary outcome measures were: (1) contact with outreach workers, (2) utilization of escorted referrals, (3) forced drinking at work, and (4) responses to GBV and GBV acceptance.

The primary and secondary outcomes were tracked and measured using self-reported data from the baseline, midline, and endline surveys. The survey questionnaires contained items on demographics and background history; entertainment work; sexual behaviors; condom use self-efficacy; HIV risk perception, testing, and treatment; STI testing and treatment; contraception use and pregnancy; GBV and inequity; substance abuse; psychological distress; linkage to health services; and exposure to the Mobile Link 
intervention. The questionnaires contained approximately 100 questions, which were either dichotomous (e.g., yes/no), categorical (e.g., type of contraception method used), ordinal (e.g., always, frequently, sometimes, and never), or a ratio (e.g., number of years working in entertainment venues). The questionnaires were adapted from validated questionnaires used in our previous research in FEWs in Cambodia. The questionnaires were created in English, translated to Khmer, and back-translated to English. The Khmer questionnaires were validated via a pilot test of $15 \mathrm{FEWs}$ with similar characteristics to the intervention participants, who were later excluded from the main surveys.

\section{Data collection}

The data collection process involved a baseline, midline, and endline questionnaire survey and weekly monitoring data. The questionnaires were administered in-person in Khmer by the Mobile LinKs female field researchers using the open-source Kobo Toolbox software installed on Android-operating tablets. Before data collection, the field researchers underwent two-day training in which the questionnaire was also pretested. Field staff connected FEWs to field researchers by making appointments with FEWs, following up the appointments, and guiding FEWs to predetermined interview locations. We conducted the baseline survey before the start of the intervention (March 2018), the midline survey at six months after baseline (November 2018), and the endline survey at 12 months after baseline (June 2019). The questionnaires took approximately 25 to 30 minutes to complete, and the field researchers were blind to the FEWs treatment arm assignment to reduce the possibility of bias.

\section{Ethical considerations}

The Mobile Link intervention engaged community and public health stakeholders to ensure that the study incorporates best practices and strong ethical standards. Due to the sensitive nature of HIV, SRH, and GBV topics presented in the surveys and questionnaires, additional steps were taken to ensure participants' safety and well-being. First, all data collectors received training related to asking sensitive questions. Second, upon obtaining informed consent, community health workers disclosed information, making clear the sensitive topics discussed in the data collection process. Third, participants were offered escorted referrals to counseling services and provided with services upon request. Participants could be connected to services in the event of an adverse outcome through the SMS/VM platform. Also, participants could leave the study at any time. Furthermore, participants' identities were kept confidential and stored securely in password-protected files. Coded identifiers were given to participants after obtaining informed consent. No participants' personal identifiers were used in analyses or report writing. Participants received $\$ 5$ in compensation and transportation reimbursement for their participation.

\section{Statistical analyses}

STATA/SE 15.1 (College Station, TX, USA) was used for statistical analyses. We tabulated participants' baseline characteristics and distributions of primary and secondary outcome variables for intervention versus control arms for the analytic sample - participants with at least two observations - using frequencies and proportions for categorical variables and means and standard deviations (SDs) for continuous variables. These characteristics were compared by group using tests of association, including 
Pearson's Chi-squared tests of homogeneity for categorical variables and paired Student's t-tests for continuous variables to ensure the balance between the study arms. We conducted both crude and cluster-adjusted pooled tests of association to account for clustering within workplace venues. Participant characteristics were then compared for the analytic sample $(n=388)$ versus non-analytic sample $(n=733)$ to assess significant differences within and between groups for those retained in the study per protocol for at least two survey assessments (i.e., analytic sample) versus those lost to follow up after the baseline assessment (i.e., non-analytic sample).

Intervention effects were assessed using multilevel mixed-effects logistic regression to model all binary outcomes accounting for within-subject correlation from taking repeated measures on the same participants over time (two-level models with observations nested within individuals). Clustered standard errors were computed to account for the similarity of characteristics and behaviors among participants in the same venues. Separate models were conducted for each primary and secondary outcome. Model fit was assessed for each outcome using the Akaike Information Criterion (AIC) and Bayesian Information Criterion (BIC).

Predictors in each simple unadjusted two-level mixed-effects logistic regression model included: group, time, and group by time interaction terms. Intervention effects for each outcome were determined by group by time interaction terms at endline with a significant $p$-value $<0.05$. Odds ratios (ORs) and $95 \%$ confidence intervals (Cls) for intervention effects at endline are displayed in Tables 2 and 3 (group by time interactions at time 3). Significant interactions indicating intervention effects were graphed using the marginsplot command (Figs. 1-3). Midline effects (significant group by time interactions at time 2) are displayed in the figures but not in the tables. For the fully adjusted primary and secondary outcome models, the following covariates were included to control for alternative explanations: entertainment job venue type, province, cohabitation, age, and education. For primary outcomes, contact with outreach workers in the last six months was also included as a covariate to assess the impact of linkage support on HIV and STI testing, contraceptive use, and condom use.

As a sensitivity analysis, we used intention-to-treat (ITT) principles for modeling primary and secondary outcomes with all participants $(n=1121)$, according to the arm to which they were assigned and then compared to the results for each outcome from the per-protocol modeling with the analytic sample. Perprotocol analyses were undertaken to assess the intervention's impact among those who actively participated in the study. Participants lost to follow-up after baseline, resulting in missing outcome data at six months, were considered non-users. ITT and per-protocol results were consistent for all outcomes regarding the direction, strength, and significance of associations. As such, only the per-protocol results are presented in the tables for ease of interpretation.

\section{Protocol adaptations}

There are several protocol deviations to note. The original protocol called for a 12-month (52 week) trial. However, due to high dropout rates at the midterm, we extended the trial to 60 weeks to recruit and enroll more participants who would have the chance to be exposed to the intervention for at least 30 weeks. 
We did not anticipate the level of loss to follow-up that occurred and, therefore, did not have a plan in place for replacement recruitment in our original protocol. We decided to recruit replacement participants at the midline by randomly selecting FEWs from our master list from the same venue and age group. In our analyses, we defined exposure as having had at least 30 weeks of exposure to the intervention.

Another deviation occurred in our group assignment plan. Initially, we planned to randomize at the entertainment venue level to conduct a cluster RCT. Before the implementation, we changed our trial design to randomize at the individual level due to the high level of movement of FEWs between venues. As a result, we modeled intervention effects using the individual rather than the venue as the analysis unit. We computed clustered standard errors based on the venue rather than including the venue as a level in the mixed-effects outcome models. Finally, we included the venue type (e.g., karaoke bar, beer garden, etc.) as a covariate in all our models.

In our protocol, we planned to send out weekly survey questions to intervention participants on various health topics. During intervention development, we heard from pilot participants that they felt reluctant to give that type of information through the phone. We were also concerned about message fatigue, privacy, and literacy and decided to omit that part of the intervention.

Finally, in our protocol, we stated that we would present an ITT analysis. Because the ITT and perprotocol findings were the same, we decided to present the per-protocol analysis for ease of interpretation.

\section{Results}

The study's participant flow diagram is depicted in Fig. 1. Before the intervention started, $3295 \mathrm{FEWs}$ were assessed for eligibility, of whom 828 FEWs did not meet the eligibility criteria, 134 declined to participate, and 325 FEWs were excluded because of other reasons. Of the included FEWs, 435 FEWs were allocated to the intervention group and $683 \mathrm{FEWs}$ to the control group. By the end of 30 weeks, $217 \mathrm{FEWs}$ in the intervention and $513 \mathrm{FEWs}$ in the control group discontinued the study and were replaced. We included 435 FEWs in the intervention and 683 FEWs in the control group in intention-to-treat analyses and 218 FEWs in the intervention and $170 \mathrm{FEWs}$ in the control group in the per-protocol analyses.

Table 1 displays characteristics of the sample stratified by intervention vs. control group with crude and cluster-adjusted pooled association tests. When accounting for clustering by the venue, there were no differences in sample characteristics by the group at baseline except a significantly lower proportion of currently married participants in the intervention than in the control group ( $19 \%$ vs. $28 \%, p=0.047)$. There were crude differences identified by province and entertainment job venue type when not accounting for clustering within venues. The intervention group had a higher proportion of participants from Battambang than the control group ( $16 \%$ vs. $9 \%, p=0.04)$. There were marginal but not significant crude differences in the proportions of intervention vs. control participants in each of the other three provinces. The intervention group had a higher proportion of participants working in karaoke bars $(67 \%$ vs. $55 \%, p=$ 0.01). In contrast, the control group had a higher proportion of participants working in beer gardens ( $12 \%$ 
vs. $22 \%, p=0.009)$. All other characteristics were successfully matched between arms with no statistically significant differences between intervention and control groups.

We compared the characteristics of the analytic (retained) vs. non-analytic (lost to follow-up) samples and identified significant baseline differences in believing that something can be done if someone experiences abuse and forced drinking at work. Participants in the analytic sample were more likely to believe that something can be done if a person experiences abuse and to report ever being forced to drink at work. No other baseline differences were identified between analytic and non-analytic samples.

Table 2 shows intervention effects at endline on primary outcomes in unadjusted and adjusted models. There was a statistically significant improvement in the frequency of self-reported condom use with nonpaying partners in the control group than the intervention group in the unadjusted model (group by time 3 OR $0.26,95 \% \mathrm{Cl} 0.08-0.83$ ). However, no significant differences between intervention and control groups over time were observed for any primary outcomes in the fully adjusted models.

As shown in Table 3, secondary outcomes with significant intervention effects at endline in unadjusted models included: contacting an outreach worker (group by time 3 OR 3.31, 95\% Cl: 1.06-10.33), receiving an escorted referral (group by time $3 \mathrm{OR} 9.51,95 \% \mathrm{Cl} 2.06-43.95$ ), and never being forced to drink at work (group by time 3 OR 4.28, 95\% Cl 1.72-10.65). Statistically significant evidence for positive intervention effects at endline was detected for the following secondary outcomes in the fully adjusted models: receiving an escorted referral (group by time 3 AOR 8.15, 95\% $\mathrm{Cl} 1.65-40.25$ ), and never being forced to drink at work (group by time 3 AOR: $3.95,95 \% \mathrm{Cl} 1.62-9.60$ ). Significant midline effects were also detected for contacting an outreach worker (group by time 2 AOR 3.29, 95\% $\mathrm{Cl} 1.28-8.47$ ) and receiving an escorted referral (group by time 2 AOR 2.86, 95\% $\mathrm{Cl} 1.09-7.52$ ). This effect was not observed at the endline for contacting an outreach worker.

Figure 2-4 show intervention effects overtime on contact with outreach workers (Fig. 2), escorted referrals (Fig. 3), and never being forced to drink at work (Fig. 4).

\section{Discussion}

Our findings suggest that the Mobile Link intervention effectively connected FEWs with outreach workers for health information and escorted referrals. However, the findings do not indicate an impact on HIV and STI testing, condom use, and contraceptive use. Reductions in forced drinking at work were significantly larger in the intervention group than in the control group. Besides, improvements on many primary and secondary outcomes in both groups might suggest that enrollment in the study had positive effects on FEWs overall, regardless of whether they were assigned to receive intervention messages.

Connecting hard-to-reach populations to prevention and treatment services through outreach workers using mobile phone messages is effective in other mHealth studies in low- and middle-income countries [27-29]. RCT evaluations of mHealth interventions have demonstrated positive changes in knowledge and attitudes but have also been limited in their ability to show changes in health outcomes [29]. In our 
theory of change, more health knowledge, paired with more contact with outreach workers, will eventually lead to increased health service use and ultimately improved health outcomes. Our study did not detect changes in health outcomes, perhaps because these changes take longer to occur. It is also possible that several trial implementation challenges may have limited ability to detect health outcomes changes, including the high loss to follow-up, which was identified as an issue for other mHealth studies in Cambodia [30-32].

Our findings indicate reductions in forced drinking at work among intervention participants. The pressure to drink alcohol at work from supervisors and peers is common for FEWs in Cambodia [25]. During our qualitative intervention development, FEWs expressed a desire to reduce alcohol consumption at work despite this pressure. Messages developed in response to this request and sent out through the Mobile Link platform advised about subtly avoiding and reducing the effect of heavy drinking. The tips included eating large meals before work, drinking lots of water in between drinks, adding lots of ice to displace the alcohol, and sharing tips to reduce pressure to drink more for tips. As alcohol use is linked to increased sexual risk-taking and violence, these findings are promising.

These findings should be interpreted with regard to study limitations. First, differential loss to follow-up was observed between intervention and control groups, and attrition was particularly high among control and replacement participants. Second, recruiting new participants to replace those lost to follow-up was conducted according to the initially intended distribution of participants per province rather than where participants were lost, resulting in an overrepresentation of control participants in smaller provinces. Third, high levels of movement between venues among FEWs led to a protocol change resulting in individual-level rather than venue-level sampling and non-random assignment to intervention and control groups. As such, the findings should be interpreted with caution. However, a balance between study arms at baseline was achieved on all primary and secondary outcomes, in both the analytic sample and full sample, suggesting that intervention and control participants were appropriately matched based on participant characteristics and health behaviors.

Despite these limitations, this study demonstrates that mHealth approaches can link hard-to-reach populations with necessary health services. Similar to the implications of meta-analysis findings of SMS interventions for antiretroviral adherence support in sub-Saharan Africa [19], messaging content may not have significant impacts on behaviors relative to linkage to healthcare workers (i.e., nurses or community health workers). However, other text-messaging interventions RCTs focused heavily on message content and with greater intensity but shorter duration than this intervention, have found significant impacts on multiple HIV-related risk behaviors and relative to live messaging communication with community health workers [31]. Client-centered messaging contents may also serve to enhance and sustain engagement in the intervention and seed motivation to connect to a community health worker for support and escorted service linkages. Effects likely depend on the population's risk, resources in context, the intervention intensity, and other intervention details.

\section{Conclusions}


Given the positive findings on service linkages for this intervention, we will consider using the Mobile Link model with other key populations in Cambodia and the region. The traditional in-person visitation model by community health workers on two-week or monthly rotations, the standard of care during this trial, may be enhanced by interventions such as the Mobile Link. This study is unique because of the extensive qualitative intervention development period. Replication of any messaging service would benefit from qualitative research to inform adaptation. Successfully linking vulnerable young women to outreach workers and escorted referrals services through their mobile phones may lead to linkages to other types of services, events, rights-based information, and behavior change messaging. Longer-term messaging and prompts of community health worker linkage have the potential to increase access to services and may impact FEWs' health outcomes in the future.

\section{Abbreviations}

AIC: Akaike Information Criterion; AOR: Adjusted odds ratio; BIC: Bayesian Information Criterion; Cl: confidence interval; FEW: Female entertainment workers; FGD: Focus group discussions; GBV: Genderbased violence; HIV: Human immunodeficiency virus infection; IDI: In-depth interviews; ITT: Intention-totreat; KIl: key informant interviews; mHealth: Mobile health; NGO: Non-governmental organizations; RCT: Randomized controlled trial; SD: Standard deviations; SIM: Subscriber identification module; SMS: Short message service; SRH: Sexual and reproductive health; STIs: Sexually transmitted infections; VM: Voice message; WMC: Women's Media Center.

\section{Declarations}

\section{Ethical approval and consent to participate}

This study was approved by the National Ethics Committee for Health Research of the Ministry of Health in Cambodia (No. 142NECHR) and the Institutional Review Board of Touro University (No. PH-0117). Written informed consent was obtained from each participant.

\section{Consent for publication}

Not applicable.

\section{Availability of data and materials}

The datasets used and/or analyzed during the current study are available from the corresponding author on reasonable request.

\section{Competing interest}

The authors declare that they have no competing interests.

\section{Authors' contributions}


CB conducted the literature review. CB, SY, and DS developed the study design. SY, PC, and ST managed the project implementation and data collection. AF and AM conducted the data analyses. $C B, A F, A M$, and SY managed the data interpretation. CB wrote the first draft of the manuscript. All authors reviewed, edited, and approved the final version of the manuscript.

\section{Funding}

This study was funded by the $5 \%$ Initiative through Expertise France (Grant No. 16SANIN210). CB received additional support from NIH Grants P30AI028697 and UL1TR000124. AEF was supported by a center grant at the University of California, Los Angeles (UCLA) Center for HIV Identification, Prevention, and Treatment Services (NIMH P30MH058107), a training grant at the UCLA Semel Institute for Neuroscience and Human Behavior (NIMH T32MH109205), and a training grant at the University of California Global Health Institute (UCGHI) at the University of California, San Francisco from the NIH Fogarty International Center (FIC D43TW009343). CB, AEF, and DS acknowledge the UCGHI Center of Expertise in Women's Health, Gender, and Empowerment for supporting the intellectual environment related to this work. SY was partially supported by the UHS-SSHSPH Integrated Research Program (USIRP) of Saw Swee Hock School of Public Health, National University of Singapore (R-608-000-247750). The funding bodies had no roles in the study design; data collection, analyses, and interpretation; and manuscript writing.

\section{Acknowledgements}

The research team would like to express gratitude for the participants who contributed their time to participate in the study. We also thank our Cambodian implementing partners, National AIDS Authority, National Center for HIV/AIDS, Dermatology and STD, and Ministry of Post and Telecommunication for their support.

\section{References}

1. Baral S, Beyrer C, Muessig K, Poteat T, Wirtz AL, Decker MR, et al. Burden of HIV among female sex workers in low-income and middle-income countries: a systematic review and meta-analysis. Lancet Infect Dis. 2012; 12(7):538-49.

2. The Joint United Nations Programme on HIV/AIDS (UNAIDS). UNAIDS guidance note on HIV and sex work. Geneva: UNAIDS; 2009.

3. Gorry J, Roen K, Reilly J. Selling yourself? The psychological impact of street sex work and factors affecting support seeking. Health Soc Care Community. 2010; 18(5):492-9.

4. Scorgie F, Chersich MF, Ntaganira I, Gerbase A, Lule F, Lo Y-R. Socio-demographic characteristics and behavioral risk factors of female sex workers in Sub-Saharan Africa: a systematic review. AIDS Behav. 2012; 16(4):920-33.

5. Argento E, Reza-Paul S, Lorway R, Lorway R, Jain J, Bhagya M, et al. Confronting structural violence in sex work: lessons from a community-led HIV prevention project in Mysore, India. AIDS Care. 2011; 
23(1):69-74.

6. International Labour Organization (ILO). Cambodia - addressing HIV vulnerabilities of indirect sex workers during the financial crisis; situation analysis, strategies and entry points for HIV/AIDS workplace education. Bangkok: ILO; 2011.

7. Brody C, Chhoun P, Tuot S, Swendeman D, Yi S. Childhood conditions, pathways to entertainment work and current practices of female entertainment workers in Cambodia: baseline findings from the Mobile Link trial. PLoS One. 2019; 14(10):e0216578.

8. Ministry of Education, Youth and Sport (MoEYS). Examining life experiences and HIV risks of young entertainment workers in four Cambodian cities. Phnom Penh: MoEYS; 2012.

9. World Bank Group. Cambodia economic update, 2018. Phnom Penh: World Bank; 2018.

10. Couture M-C, Sansothy N, Sapphon V, Phal S, Sichan K, Stein E, et al. Young women engaged in sex work in Phnom Penh, Cambodia, have high incidence of HIV and sexually transmitted infections, and amphetamine-type stimulant use: new challenges to HIV prevention and risk. Sex Transm Dis. 2011; 38(1):33-9.

11. Yi S, Tuot S, Chhoun P, Pal K, Tith K, Brody C. Factors associated with induced abortion among female entertainment workers: a cross-sectional study in Cambodia. BMJ Open. 2015; 5(7):e007947.

12. Wieten CW, Chhoun P, Tuot S, Brody C, Yi S. Gender-based violence experiences among female entertainment workers in Cambodia: a cross-sectional study. J Interpers Violence. 2020. (In press).

13. Platt L, Grenfell P, Meiksin R, Elmes J, Sherman SG, Sanders T, et al. Associations between sex work laws and sex workers' health: A systematic review and meta-analysis of quantitative and qualitative studies. PLoS Med. 2018;15(2):e1002680.

14. United Nations Development Programme (UNDP), Asia-Pacific Regional Centre. Sex work and the law in Asia and the Pacific: laws, HIV and human rights in the context of sex work. Bangkok: UNDP, AsiaPacific Regional Centre; 2012.

15. Human Rights Watch. Off the streets: arbitrary detention and other abuses against sex workers in Cambodia. New York: Human Rights Watch; 2010.

16. Page K, Stein E, Sansothy N, Evans J, Couture M-C, Sichan K, et al. Sex work and HIV in Cambodia: trajectories of risk and disease in two cohorts of high-risk young women in Phnom Penh, Cambodia. BMJ Open. 2013; 3(9):e003095.

17. Agarwal S, Perry HB, Long L-A, Labrique AB. Evidence on feasibility and effective use of mHealth strategies by frontline health workers in developing countries: systematic review. Trop Med Int Health. 2015; 20(8):1003-14.

18. L'Engle KL, Vahdat HL, Ndakidemi E, Lasway C, Zan T. Evaluating feasibility, reach and potential impact of a text message family planning information service in Tanzania. Contraception. 2013; 87(2): 251-6.

19. Mbuagbaw L, van der Kop ML, Lester RT, Thirumurthy H, Pop-Eleches $C$, Ye C, et al. Mobile phone text messages for improving adherence to antiretroviral therapy (ART): an individual patient data metaanalysis of randomised trials. BMJ Open. 2013; 3(12):e003950. 
20. Chib A, van Velthoven $\mathrm{MH}$, Car J. mHealth adoption in low-resource environments: a review of the use of mobile healthcare in developing countries. J Health Commun. 2015; 20(1):4-34.

21. Colaci $D$, Chaudhri $S$, Vasan A. mhealth interventions in low-income countries to address maternal health: a systematic review. Ann Glob Health. 2016; 82(5):922-35.

22. Gurman TA, Rubin SE, Roess AA. Effectiveness of mhealth behavior change communication interventions in developing countries: a systematic review of the literature. J Health Commun. 2012; 17(Suppl 1):82-104.

23. Chhoun P, Kaplan KC, Wieten C, Jelveh I, Lienemann M, Tuot S, et al. Using participatory methods to build an mHealth intervention for female entertainment workers in Cambodia: the development of the Mobile Link project. mHealth. 2019; 5:24.

24. Brody C, Tuot S, Chhoun P, Swendeman D, Kaplan KC, Yi S. Mobile Link-a theory-based messaging intervention for improving sexual and reproductive health of female entertainment workers in Cambodia: study protocol of a randomized controlled trial. Trials. 2018; 19(1):235.

25. Brody C, Reno R, Chhoun P, Kaplan K, Tuot S, Yi S. Female entertainment workers and condom use negotiation in post-100\% condom use era Cambodia. Arch Sex Behav. 2020; 49(8):3065-74.

26. Ippoliti NB, L'Engle K. Meet us on the phone: mobile phone programs for adolescent sexual and reproductive health in low-to-middle income countries. Reprod Health. 2017; 14(1):11.

27. Smith C, Ngo TD, Gold J, Edwards P, Vannak U, Sokhey L, et al. Effect of a mobile phone-based intervention on post-abortion contraception: a randomized controlled trial in Cambodia. Bull World Health Organ. 2015; 93(12):842-50A.

28. Rokicki S, Cohen J, Salomon JA, Fink G. Impact of a text-messaging program on adolescent reproductive health: a cluster-randomized trial in Ghana. Am J Public Health. 2017; 107(2):298-305.

29. Bullen PAB. Operational challenges in the Cambodian mHealth revolution. J Mob Technol Med. 2013; 2(2):20-3.

30. Chhim S, Chhea C, Sopheab H, Mathers BM, Kaldor J, Evans JL, et al. Proportion and predictors of loss to follow-up in a longitudinal cohort study of female entertainment and sex workers in Cambodia. Int J STD AIDS. 2018; 29(13):1295-304.

31. Reback CJ, Fletcher JB, Swendeman DA, Metzner M. Theory-based text-messaging to reduce methamphetamine use and HIV sexual risk behaviors among men who have sex with men: automated unidirectional delivery outperforms bidirectional peer interactive delivery. AIDS Behav. 2019; 23(1):37-47.

32. Brody C, Kaplan KC, Tuot S, Chhoun P, Farr C, Swendeman D, et al. "We cannot avoid drinking": Alcohol use among female entertainment workers in Cambodia. Subst Use Misuse. 2020; 55(4):60212.

\section{Tables}


Intervention effects on primary outcomes among the analytic sample, unadjusted and adjusted models $(n=989)$

\begin{tabular}{|c|c|c|}
\hline & $\begin{array}{l}\text { Intervention } \\
\text { group } \\
(n=218)\end{array}$ & $\begin{array}{l}\text { Control } \\
\text { group } \\
(n=170)\end{array}$ \\
\hline & $n(\%)$ & $n(\%)$ \\
\hline \multicolumn{3}{|l|}{ Demographics } \\
\hline Age in years (mean, SD) & $24.7(3.8)$ & $\begin{array}{l}24.5 \\
(4.0)\end{array}$ \\
\hline Years of schooling attained (mean, SD) & $6.2(3.0)$ & $\begin{array}{l}6.3 \\
(3.0)\end{array}$ \\
\hline \multicolumn{3}{|l|}{ Marital status } \\
\hline Currently married ${ }^{*}$ & $42(19.3)$ & $\begin{array}{c}48 \\
(28.2)\end{array}$ \\
\hline Previously married (widowed, divorced, or separated) & $81(37.2)$ & $\begin{array}{c}55 \\
(32.4)\end{array}$ \\
\hline Never married & $95(43.6)$ & $\begin{array}{c}67 \\
(39.4)\end{array}$ \\
\hline \multicolumn{3}{|l|}{ Province } \\
\hline Phnom Penh & $86(39.4)$ & $\begin{array}{c}51 \\
(30.0)\end{array}$ \\
\hline Battambang* & $35(16.1)$ & $15(8.8)$ \\
\hline Banteay Meanchey & $52(23.8)$ & $\begin{array}{c}55 \\
(32.4)\end{array}$ \\
\hline Siem Reap & $45(20.6)$ & $\begin{array}{c}49 \\
(28.8)\end{array}$ \\
\hline Poor as a child (Multidimensional Childhood Poverty Scale score $\geq 3$ ) & $190(87.2)$ & $\begin{array}{l}145 \\
(85.3)\end{array}$ \\
\hline Weekly income in USD (mean, SD) & $\begin{array}{l}277.0 \\
(206.2)\end{array}$ & $\begin{array}{l}272 \cdot 4 \\
(167.0)\end{array}$ \\
\hline \multicolumn{3}{|l|}{ Entertainment job venue type } \\
\hline Karaoke bar ${ }^{*}$ & $146(67.0)$ & $\begin{array}{c}93 \\
(54.7)\end{array}$ \\
\hline Beer garden $^{\star}$ & $26(11.9)$ & $\begin{array}{l}37 \\
(21.8)\end{array}$ \\
\hline Other (e.g., massage, dance club, or freelance in streets/parks) & $46(21.1)$ & 40 \\
\hline
\end{tabular}


Had sex with partner in exchange for money or gifts, last 3 months $60(41.1)$

$(n=146, n=170)$

Type of message received

Short message service

$99(45.4)$

64

Voice message

$119(54.6)$

106

Health outcomes

Tested for HIV in last six months $(n=176, n=150)$

$113(64.2)$

95

Tested for STIs when most recently showed symptoms ( $n=112$, $n=71)$

$27(24.1) \quad(26.8)$

Uses modern contraceptive to prevent pregnancy

$68(31.2)$

Always uses condom with non-paying partners

$43(71.1)$

31

Always uses condom with paying clients $(n=60, n=40)$

$23(22.6)$

Frequency of forced drinking at work

Never

$123(56.4)$

111

Less than monthly

$16(7.3)$

$13(7.6)$

Monthly

28 (12.8)

$15(8.8)$

Weekly

51 (23.4)

31

(18.2)

Gender-Based Violence Acceptance Scale score (mean, SD) ( $n=26, n=74)$

(Range 0-16)

4.4 (3.7)

4.5

Believes you cannot do anything if you or someone you know experiences physical or sexual abuse

$60(27.5)$

Outreach worker contact only, last six months

$20(9.2)$

(27.6)

Number of times contacting outreach worker in the last six months

Never

$20(46.5)$

21

(58.3)

Once

$11(25.6)$

6

Two to four times

11 (25.6) 
Five or more times

Received escorted referral for HIV/STI $(n=23, n=23)$

Received escorted referral for vaginal health $(n=23, n=23)$

Notes: HIV: Human immunodeficiency virus; SD: Standard deviation; STI: Sexually transmitted infection.

*Indicates a significant difference between intervention and control arms at baseline $(p<0.05)$.
$1(2.8)$

$7(30.4)$

$14(60.9)$
4

16

Table 2

Intervention effects on primary outcomes among the analytic sample, unadjusted and adjusted models $(n=989)$

\begin{tabular}{|c|c|c|}
\hline & OR (95\% Cl) & AOR $(95 \% \mathrm{Cl})$ \\
\hline Tested for HIV, last six months $(1=$ Yes, $0=$ No $)(n=887)$ & $\begin{array}{l}0.45(0.18- \\
1.13)\end{array}$ & $\begin{array}{l}0.40(0.16- \\
1.04)\end{array}$ \\
\hline $\begin{array}{l}\text { Tested for STIs, most recent symptoms }(1=\text { Yes, No }=0)(n= \\
\text { 394) }\end{array}$ & $\begin{array}{l}1.36(0.25- \\
7.50)\end{array}$ & $\begin{array}{l}1.20(0.21- \\
7.00)\end{array}$ \\
\hline $\begin{array}{l}\text { Uses modern contraceptive to prevent pregnancy }(1=\text { Yes, No }= \\
0)(n=989)\end{array}$ & $\begin{array}{l}1.06(0.37- \\
3.02)\end{array}$ & $\begin{array}{l}0.99(0.35- \\
2.77)\end{array}$ \\
\hline $\begin{array}{l}\text { Always uses condom with non-paying partners }(1=\text { Yes, No }=0) \\
(n=586)\end{array}$ & $\begin{array}{l}0.26(0.08- \\
0.83)^{*}\end{array}$ & $\begin{array}{l}0.50(0.16- \\
1.58)\end{array}$ \\
\hline $\begin{array}{l}\text { Always uses condom with paying clients }(1=\text { Yes, No }=0)(n= \\
242)\end{array}$ & $\begin{array}{l}1.77(0.14- \\
22.74)\end{array}$ & $\begin{array}{l}1.17(0.08- \\
17.64)\end{array}$ \\
\hline \multicolumn{3}{|c|}{$\begin{array}{l}\text { Notes: AOR: Adjusted odds ratio; Cl: Confidence interval; HIV: Human immunodeficiency virus; OR: } \\
\text { Odds ratio; STI: Sexually transmitted infection. }\end{array}$} \\
\hline \multicolumn{3}{|c|}{$\begin{array}{l}\text { Adjusted models included venue type, province, cohabitation, age, education, and outreach worker } \\
\text { contact. }\end{array}$} \\
\hline \multicolumn{3}{|c|}{ *Indicates a significant difference between intervention and control arms at endline $(p<0.05)$. } \\
\hline
\end{tabular}


Table 3

Intervention effects on secondary outcomes among analytic sample in unadjusted and adjusted models $(n=989)$

\begin{tabular}{|c|c|c|}
\hline & OR $(95 \% \mathrm{Cl})$ & AOR $(95 \% \mathrm{Cl})$ \\
\hline Outreach worker contact, last six months $(1=$ Yes, $0=$ No $)$ & $\begin{array}{l}3.31(1.06- \\
10.33)^{\star}\end{array}$ & $2.82(0.93-8.55)$ \\
\hline Escorted referral, last six months $(1=$ Yes, $0=$ No $)$ & $\begin{array}{l}9.51(2.06- \\
43.95)^{* *}\end{array}$ & $\begin{array}{l}8.15(1.65- \\
40.25)^{\star \star}\end{array}$ \\
\hline $\begin{array}{l}\text { Forced drinking at work, last three months }(1=\text { Never, } 0= \\
\text { Ever) }\end{array}$ & $\begin{array}{l}4.28(1.72- \\
10.65)^{* *}\end{array}$ & $\begin{array}{l}3.95(1.62- \\
9.60)^{\star *}\end{array}$ \\
\hline $\begin{array}{l}\text { Believes you can do something if experience abuse }(1=\text { Yes, } \\
0=\text { No) }\end{array}$ & $0.65(0.27-1.59)$ & $0.68(0.28-1.62)$ \\
\hline $\begin{array}{l}\text { Gender-based violence }(1=\text { High } / \text { moderate, } 0=\text { Low })(n= \\
701)\end{array}$ & $0.81(0.25-2.59)$ & $0.86(0.28-2.68)$ \\
\hline \multicolumn{3}{|c|}{ Notes: AOR: Adjusted odds ratio; Cl: Confidence interval; OR: Odds ratio. } \\
\hline \multicolumn{3}{|c|}{ Adjusted models include venue type, province, cohabitation, age, and education. } \\
\hline \multicolumn{3}{|c|}{ *Indicates a significant difference between intervention and control arms at endline $(p<0.05)$. } \\
\hline \multicolumn{3}{|c|}{${ }^{* *}$ Indicates a significant difference between intervention and control arms at endline $(p<0.01)$. } \\
\hline
\end{tabular}

\section{Figures}




\section{Enrollment}

Assessed for eligibility ( $n=3295)$

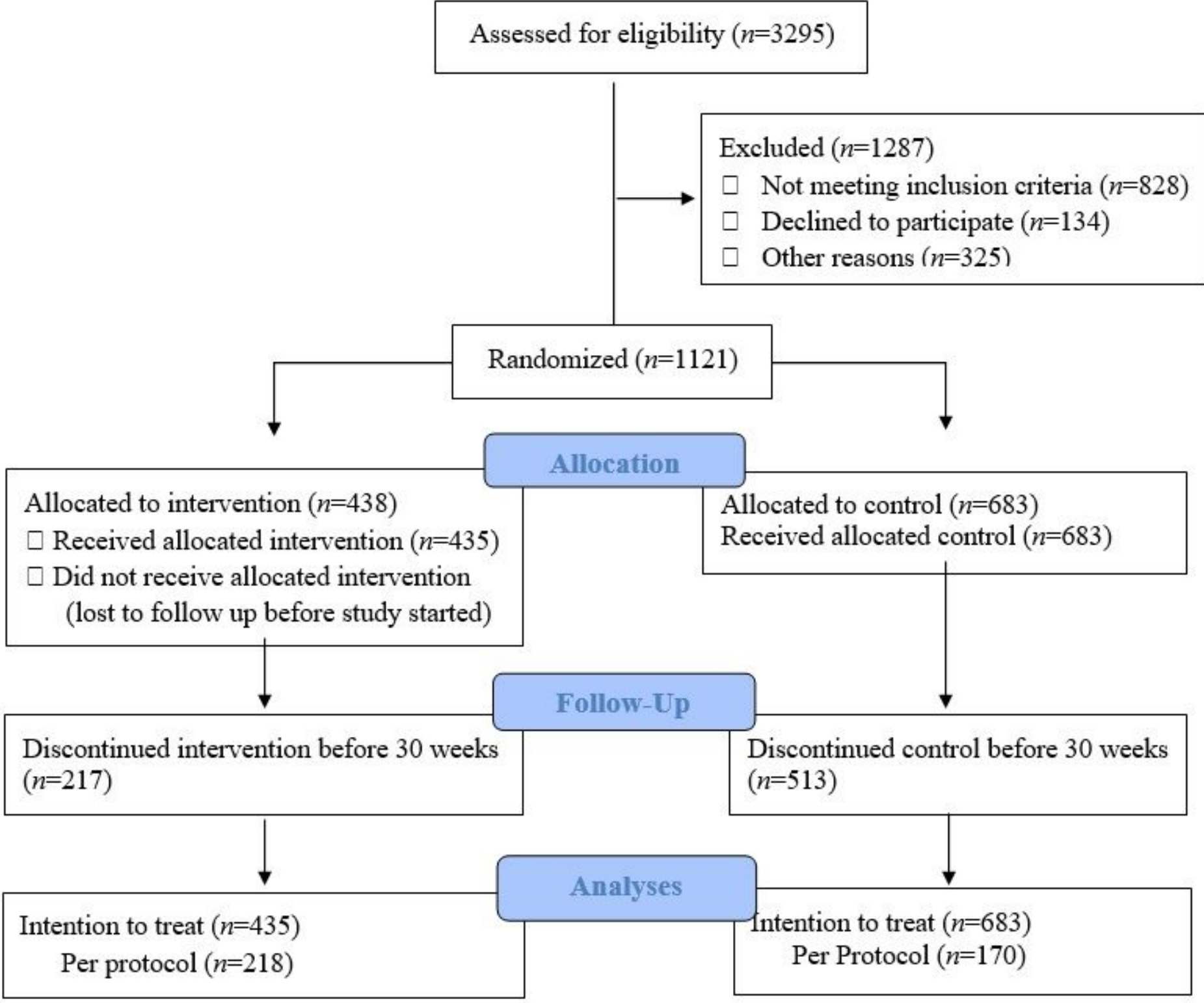

Figure 1

Participant flow diagram 
Timepoint by Treatment Group

Predictive Margins (Outreach worker contact, last six months)
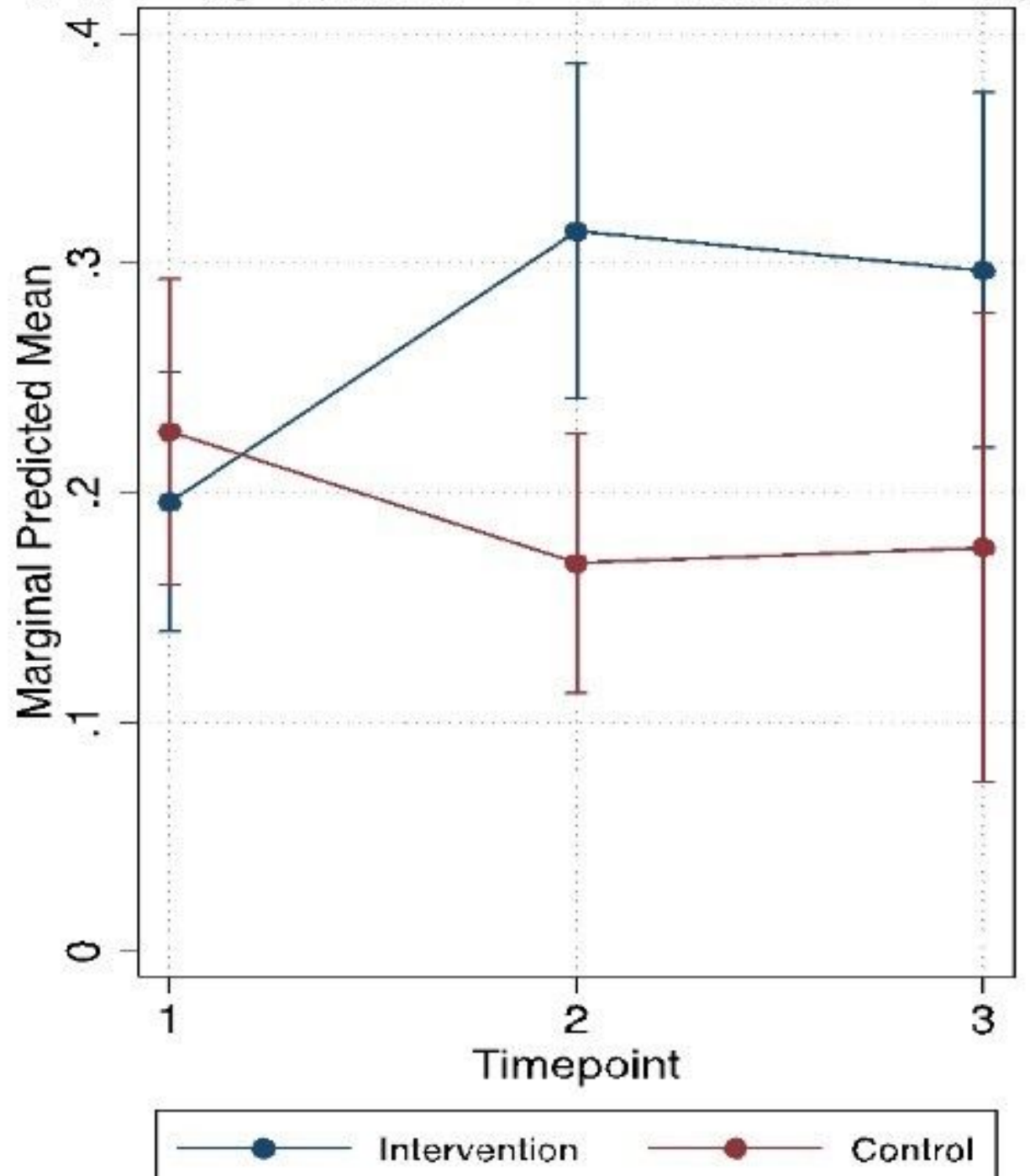

Figure 2

Intervention effects on outreach worker contact by timepoint 
Timepoint by Treatment Group

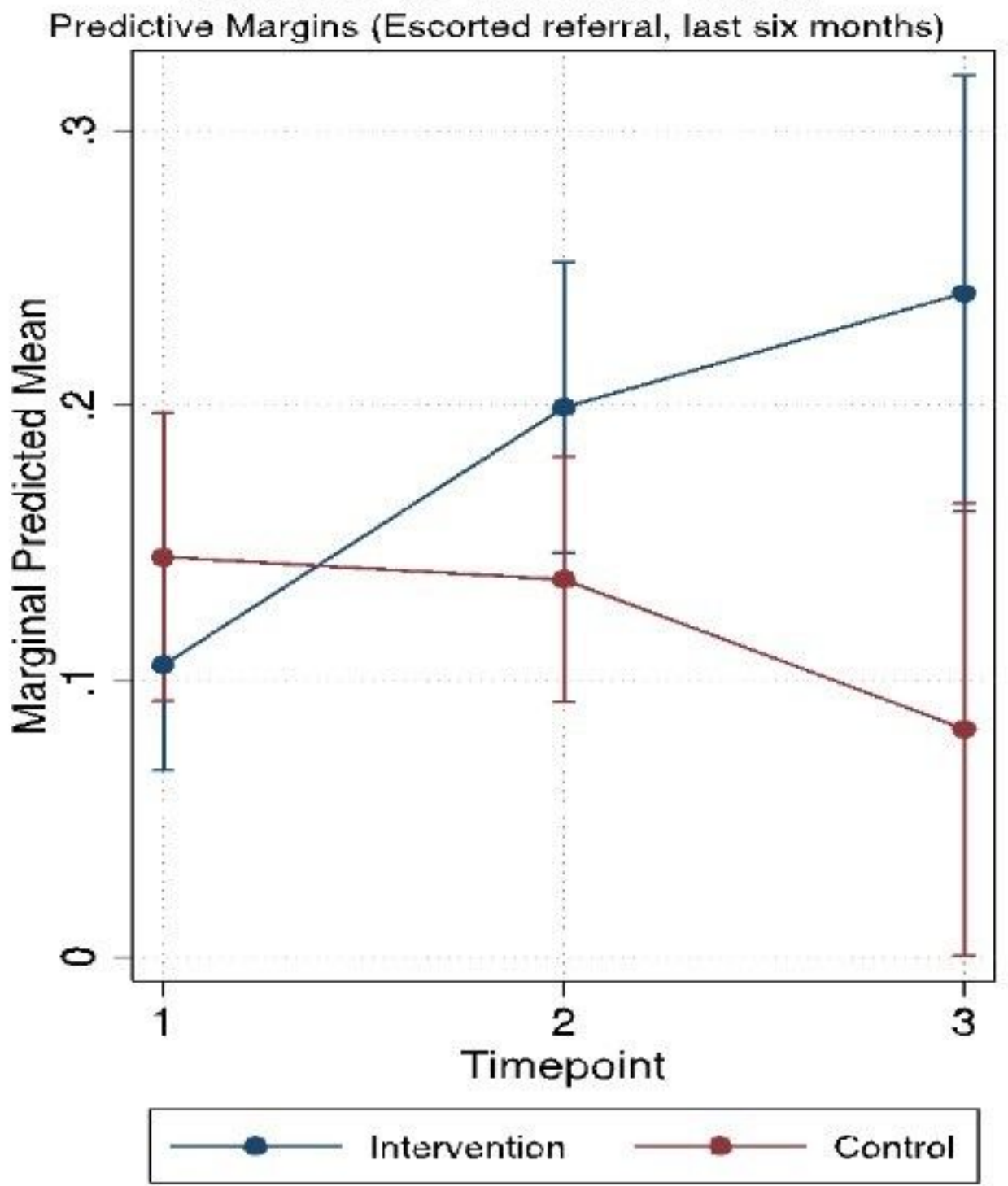

Figure 3

Intervention effects on escorted referrals by timepoint 


\section{Timepoint by Treatment Group}

Predictive Margins (Forced drinking at work when you had no desire to drink)

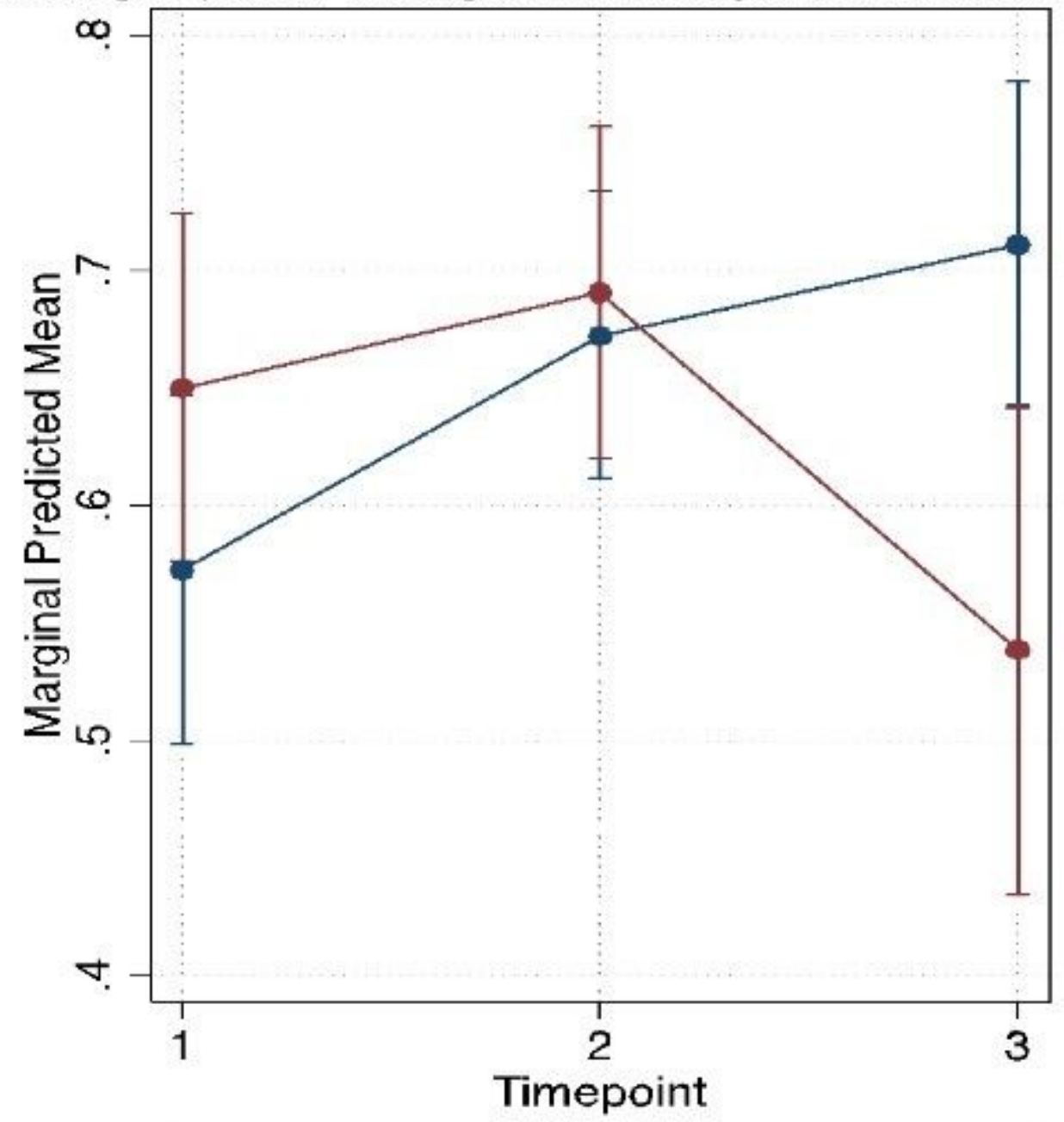

Intervention

Control

\section{Figure 4}

Intervention effects on never being forced to drink at work by timepoint

\section{Supplementary Files}

This is a list of supplementary files associated with this preprint. Click to download.

- CONSORTChecklist.doc 